\title{
La décentralisation en milieu urbain : l'arrondissement ou le quartier ? Le cas de la ville de Québec
}

\author{
Yvon Leclerc ${ }^{1}$ et Jacques Fiset ${ }^{2}$
}

Le rapport de force entre une administration gouvernementale centralisée et des administrations locales qui réclament plus de responsabilités a, pour ainsi dire, toujours existé. Il faut reconnaître, cependant, que ce rapport a lentement évolué, chez nous, depuis la Révolution tranquille et que le point de départ a été la création du ministère de l'Éducation. Car, aussi longtemps que l'élite scolarisée se concentrait dans les officines du gouvernement central et qu'elle alimentait le doute sur la capacité des acteurs locaux et des élus municipaux à assumer des missions d'intérêt public, le gouvernement leur accordait des miettes de responsabilité. Exception faite des grandes villes, et encore.

Mais depuis, le système d'éducation a fait sentir ses effets (Lévesque, $2003: 170^{3}$. L'empowerment des individus se manifeste dans les organisations locales, dans la vie communautaire et dans la vie politique. Plus personne n'oserait prétendre que la science et la connaissance se concentrent uniquement dans l'administration centrale. À Québec comme à Ottawa. On n'y fait pas moins ni plus d'erreurs que dans les organisations locales. Les missions de service public sont repensées et on cherche à les redistribuer aux échelons les plus efficaces.

\section{Un engagement gouvernemental : la décentralisation}

De son côté, le gouvernement élu le 14 avril dernier, à Québec, s'est engagé à procéder à une décentralisation importante de responsabilités et de moyens vers les élus municipaux. Son programme propose la création de la Conférence régionale des élus où se retrouveraient les préfets des municipalités régionales de comté (MRC), les maires des villes de centralité et les députés de l'Assemblée nationale. Bien que le mandat de ces conférences régionales ne soit pas encore défini, il faut en saluer l'intention et appuyer la démarche qui s'inscrit dans le cours normal de l'évolution de nos sociétés occidentales.

Des questions se posent relativement à la mise à place de ces conférences régionales. Par exemple, qu'adviendra-t-il de la Politique de soutien au développement local et régional? Les lois municipales actuelles ne devront-elles pas être modifiées substantiellement pour accueillir les mandats de décentralisation? D'autres questions concernent la Loi sur l'aménagement et l'urbanisme et les amendements successifs pour permettre à la MRC de remplir sa mission. Aussi longtemps que la MRC assurait l'élaboration et la révision du schéma d'aménagement, elle agissait avant tout comme un organisme de concertation qui visait le rapprochement rural urbain. Mais dès lors que la MRC devrait assumer la responsabilité du développement, la procédure actuelle de prise de décision, la répartition des quote-part ainsi que le processus d'imputabilité ne devront-ils pas être revus en fonction de la ou des nouvelles responsabilités qui lui seraient accordées?

Il s'agit là d'un immense chantier puisque chaque région et chaque MRC ont leurs particularités. Il s'agit quand même d'un beau risque, car le découpage territorial des MRC s'est acquis une reconnaissance identitaire aujourd'hui peu contestée. Il faut aussi composer avec une tendance qui émerge de 
l'évolution des dernières années et qui semble s'imposer : transformer la MRC en une seule administration municipale. Une ville, une MRC. C'est le cas de la MRC Rouyn-Noranda et de celle des Iles-de-laMadeleine, et ce scénario se discute dans de nombreuses autres MRC.

\section{En milieu urbain}

Dans les grandes villes, la situation a évolué plus rapidement qu'à l'échelon de la MRC. L'audace et la volonté politique ont défini le calendrier des événements. Il faut reconnaître au gouvernement précédent un courage politique certain pour avoir fusionné d'un trait de plume les villes des communautés urbaines de Montréal et de Québec et pour avoir radicalement changé le paysage urbain des sept autres agglomérations urbaines de plus de 100000 habitants que compte le Québec.

Et comme, au Québec, on ne fait jamais les choses comme ailleurs, les fusions ne pouvaient pas se faire ici comme à Toronto ou à Winnipeg. Chaque chose a son contraire et comme le mot « défusion » n'existait pas, on l'a inventé. La résistance des citoyens de certaines villes fusionnées a été et est encore très forte, malgré les exemples probants comme Laval et Beauport qui sont nés de fusions antérieures qui ont porté fruit.

Là encore, des questions se posent. La résistance des « défusionnistes » ne pourrait-elle pas conduire à l'invention de villes qui seraient mieux arrimées sur les besoins de leurs citoyens et citoyennes? Se pourrait-il que cette résistance ne soit que l'expression de la volonté des contribuables de jouer un rôle plus grand dans l'évolution de leur milieu de vie? Se pourrait-il que les résidents de Ville St-Laurent ou de Beauport souhaitent exercer une plus grande part de responsabilité dans les affaires de leur cité ?

Mieux encore, se pourrait-il que la résistance de certains citoyens des villes fusionnées produise une sorte de point de rencontre entre la capacité de gestion des communautés locales qui composent ces villes et la décentralisation des responsabilités qui leur seraient confiées? Pour tenter de répondre à ces questions, nous avons examiné le cas de la ville de Québec.

\section{La ville de Québec}

Rappelons que la nouvelle ville de Québec est composée des treize municipalités qui formaient auparavant la Communauté urbaine de Québec. Ce qui ne veut pas dire que la ville soit divisée en treize arrondissements, car les villes de la communauté urbaine comptaient des populations de taille très inégale.

\section{L'arrondissement}

La nouvelle ville est maintenant subdivisée en huit arrondissements qui visent l'équilibre de la population entre chaque arrondissement et pour une raison toute simple : l'arrondissement se veut d'abord une partie ou une division géographique de la ville où se trouvent des services d'usage fréquent, ce qui évite à ses résidents de se déplacer sur des distances importantes. On pense, par exemple, aux bibliothèques, aux équipements de loisirs, à l'émission des permis, etc. En somme, l'arrondissement rend la vie plus facile aux contribuables.

Par contre, en examinant la carte des arrondissements de la ville, on constate rapidement que cette aire de service est encore un peu désincarnée Les repères qui servent à enraciner le sentiment d'appartenance des populations locales n'ont pas encore forgé une identité, une personnalité propre à l'arrondissement. Par exemple, l'arrondissement de la Cité inclut des quartiers aussi différents que le Vieux Québec, Saint-Jean-Baptiste, Montcalm, Cap-Blanc, Saint-Roch, Saint-Sauveur ou Saint-Sacrement. Quiconque connaît un tant soit peu la ville de Québec conviendra qu'il y a un monde de différences entre ces quartiers de la même ville et du même arrondissement. Pour forger un sentiment d'appartenance à un tel arrondissement, il faudra beaucoup plus que la distribution des services de la ville; les résidents devront vivre des occasions de se côtoyer, de se mobiliser, de célébrer des moments heureux ou malheureux, bref, il faudra que ces personnes apprennent à construire ensemble leur arrondissement. Ce qui n'est pas encore le cas aujourd'hui. 


\section{Le quartier}

En réalité, l'arrondissement constitue un regroupement de quartiers, de milieux de vie. À Québec, les huit arrondissements comptent 37 quartiers. Nous nous attarderons à l'un d'eux, le quartier SaintSauveur, pour illustrer la différence entre l'arrondissement et le quartier.

L'examen de la carte du quartier fait apparaître toute l'importance de son histoire et de son évolution. Remontons le temps jusqu'au régime français où le quartier n'était au fond qu'un lieu de transit du cheptel de la ferme de la congrégation des Récollets. Le troupeau longeait la rivière Saint-Charles qui serpente le territoire d'est en ouest pour se rendre paître dans les pâturages de la plaine qui se situait au bas de la Côte Saint-Sacrement. Ce sentier est devenu aujourd'hui la rue Saint-Vallier, artère vitale du quartier.

Les habitants du secteur perçoivent les limites de leur quartier comme s'il était entouré de barrières érigées par la géographie. Plus psychologiques que réelles, ces barrières n'en donnent pas moins l'effet de frontières urbaines. À Saint-Sauveur, ces limites commencent, à l'est, par le boulevard Langelier construit vers les années 1880 pour servir de coupefeu entre les quartiers Saint-Roch et Saint-Sauveur à la suite des feux qui avaient dévasté le secteur en 1866. On avait donc rasé un pâté de maisons pour faire le boulevard (comme Haussmann, à Paris, à la même époque !). Faut-il s'étonner que le boulevard soit perçu comme un mur par les habitants des quartiers Saint-Roch et Saint-Sauveur?

\section{En réalité, l'arrondissement constitue un regroupement de quartiers, de milieux de vie. À Québec, les huit arrondissements comptent $\mathbf{3 7}$ quartiers.}

Au sud, c'est la falaise qui délimite la haute de la basse-ville; à l'ouest, c'est la rue Saint-Sacrement et au nord, la rivière Saint-Charles. On note l'importance des voies de transport routier, fluvial ou ferroviaire dans la construction de l'esprit de quartier.
$\mathrm{Au}$ fil des ans et des constructions domiciliaires, six milieux de vie sont apparus et se sont concrétisés autour de quelques pôles principaux dont la paroisse, l'église, la caisse populaire et l'épicerie ou le magasin général. C'est à travers l'évolution de ces milieux de vie que s'est forgée et se forge encore l'identité d'un quartier. Par exemple, de six écoles primaires on est passé à trois, toutes situées dans l'axe de la rue Saint-Vallier. Des six caisses populaires, il en reste deux, situées dans le même axe. On a fermé des paroisses pour n'en garder que deux dont celle de Saint-Sauveur, la plus ancienne. L'activité commerciale s'est peu à peu concentrée le long de la rue Saint-Vallier, cœur historique du quartier. Si bien que l'esprit de clocher se transforme tout naturellement en esprit de quartier, surtout lorsqu'on passe d'une génération à l'autre.

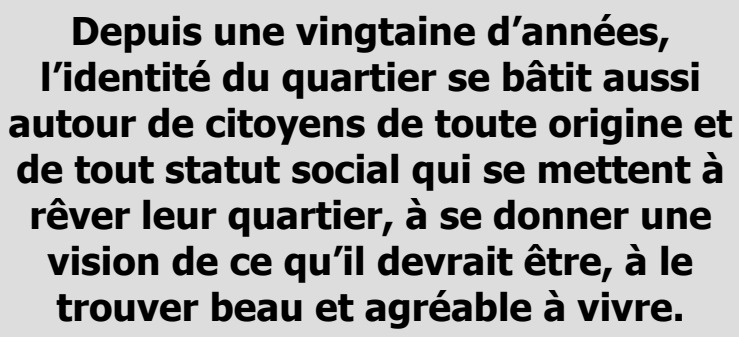

Ce sentiment d'appartenance a mis plusieurs décennies à se modeler. Il s'est forgé dans les rapports de la vie quotidienne, dans la mobilisation autour de causes communes, dans l'opposition à des décisions provenant de la ville ou du gouvernement central, etc. Et depuis une vingtaine d'années, l'identité du quartier se bâtit aussi autour de citoyens de toute origine et de tout statut social qui se mettent à rêver leur quartier, à se donner une vision de ce qu'il devrait être, à le trouver beau et agréable à vivre. Le quartier est donc un lieu de mobilisation citoyenne pour la défense comme pour la promotion des intérêts de ses habitants.

\section{Le Conseil de quartier}

C'est ici que le quartier devient un espace à considérer dans une perspective de décentralisation. On ne réfère pas seulement à la décentralisation de services municipaux, mais à toute action, décision ou programme qui concerne la qualité de vie du milieu. 
Pour bien comprendre, il est important de faire un bref retour sur l'esprit de la démocratie municipale.

D'abord, le Code municipal et la Loi des cités et villes sont des lois dites permissives. Entendre par là que tout ce qui est permis est dans la loi et ce qui n'est pas dans la loi est défendu. On réalise l'ampleur du pouvoir de l'Assemblée nationale sur les administrations municipales! Les grandes villes, heureusement, disposent de plus de moyens mais pas vraiment de plus de latitude.

Ensuite, la démocratie municipale, outre l'élection à tous les quatre ans, se résume à un exercice d'opposition. Si un groupe de citoyens s'oppose à une décision municipale, la loi prévoit l'ouverture du registre et, à terme, la tenue d'un référendum. Rien, par contre, n'existe pour permettre aux contribuables qui souhaitent la réalisation de projets touchant la vie de leur quartier de les faire valoir. Dans le monde municipal, la démocratie de participation existe donc pour s'opposer et non pour permettre d'avancer des idées ou de déposer des projets.

C'est un parti politique, le Rassemblement populaire, qui eut l'idée de proposer un mécanisme de construction plutôt que d'opposition. Ce mécanisme, c'est le Conseil de quartier. Son rôle ? Mener des consultations sur quelques sujets précis comme les règlements de zonage et les projets municipaux d'immobilisation (quand on sait à quel point le béton peut forger ou briser la vie d'un quartier). Mais le rôle le plus significatif et le plus original consiste à offrir aux citoyens et aux citoyennes un pouvoir d'initiative sur tous les aspects de la vie du quartier susceptibles d'en améliorer la qualité.

\section{Le rôle le plus significatif et le plus original du Conseil de quartier consiste à offrir aux citoyens et aux citoyennes un pouvoir d'initiative sur tous les aspects de la vie du quartier susceptibles d'en améliorer la qualité.}

Il est important de noter que le Conseil de quartier est un organisme à but non lucratif, soutenu financièrement par la ville, et que ses membres sont élus par l'assemblée générale annuelle des résidents du quartier qui pourrait même décider de ne pas se doter d'un conseil de quartier. Quant au conseil d'arrondissement, il est formé exclusivement des conseillers municipaux du secteur.

On peut donc considérer que le Conseil de quartier est un dispositif de participation citoyenne qui offre aux habitants du quartier un pouvoir d'initiative qui leur permet d'agir sur les besoins, les problèmes, les solutions du milieu, sans limitation des secteurs d'activité (économie, culture, environnement, loisirs, sécurité, etc. ).

L'effet premier de la mise en place du Conseil de quartier a été de donner confiance aux contribuables dans l'administration municipale parce que la ville respecte les avis du Conseil de quartier dans une proportion de $95 \%$. Les habitants du quartier sentent qu'ils ont leur mot à dire sur des décisions qui concernent leur environnement de vie et peuvent faire des suggestions et ne s'en privent pas. Par exemple, ce sont eux qui ont demandé les patrouilles à pied dans certaines rues, la présence de brigadiers scolaires à certaines heures, la pose de signaux d'arrêt ou le changement de sens de certaines rues, etc. Des décisions qui appartiennent bien davantage aux résidents qu'aux fonctionnaires de la ville, si compétents soient-ils.

L'analyse des priorités établies dans plusieurs autres quartiers de la ville vont dans le même sens que celles des habitants du quartier Saint-Sauveur. On s'y préoccupe d'abord de la paix, de la quiétude et de la sécurité du quartier. On veut un quartier où il fait bon vivre. On s'inquiète de la vie des enfants et de l'accès aux écoles, des personnes âgées et de l'accès aux soins de première ligne; on livre la lutte à des activités illégales comme les piqueries et la prostitution de rue; on fait des suggestions à la ville sur l'environnement physique du quartier, les loisirs, l'aménagement de parcs et de coins de verdure, etc. On relève aussi de l'analyse de ces priorités que si la ville accepte, dans une proportion si élevée, les suggestions des conseils de quartier, c'est avant tout lié au fait que les assemblées générales choisissent de s'occuper de responsabilités qui concernent la vie 
du quartier et ne cherchent pas à régler les problèmes de la ville ou des autres quartiers.

Une idée heureuse et à retenir de l'expérience des conseils de quartier concerne l'élaboration d'un Plan de quartier ${ }^{4}$. La réalisation d'un plan de quartier constitue l'instrument de choix de participation à la démocratie municipale. Le plan exige la mobilisation des habitants du quartier. On rappelle l'histoire et l'évolution du quartier afin de construire l'avenir en respectant le passé. On élabore ensuite le plan en établissant les priorités et en faisant les arbitrages pour en fixer l'ordre de réalisation. C'est ainsi que le Plan triennal d'immobilisation de la ville et de l'arrondissement est fortement influencé par le plan de quartier.

\section{Conclusion}

Les maires de Québec et de Montréal annonçaient, à la mi-août, un vaste plan d'humanisation de l'administration municipale afin de décourager les nostalgiques de l'époque où l'Hôtel de ville se situait sur la rue voisine. Dans les deux villes, les arrondissements seront des lieux de services de proximité qui auront pour effet de décongestionner l'administration centrale de la ville. Appliqué systématiquement, le principe de subsidiarité devrait conduire à délocaliser dans les arrondissements le plus grand nombre possible de services qui touchent directement la qualité de vie des gens. Que les centralisateurs se le tiennent pour dit !

Pour nous, humaniser l'administration municipale signifie une chose et son contraire. D'abord, l'administration centrale ne devrait conserver aucun service de proximité qui serait mieux servi dans l'arrondissement et dans le quartier. L'arrondissement et le quartier, c'est la première ligne, là où vit le citoyen. La répartition des missions doit en tenir compte. $A$ contrario, les arrondissements et les quartiers ne devraient pas assumer des responsabilités qui auraient pour effet de faire renaître la concurrence effrénée qui a stérilisé le développement de nos grandes villes.

La capacité d'action des individus et des communautés locales est aujourd'hui plus grande que jamais. Les villes qui misent sur les forces des habitants de leurs quartiers partent gagnantes, car l'empowerment des individus et des communautés constitue l'ingrédient premier du développement harmonieux et dynamique d'un milieu. Le Conseil de quartier répond bien à cette condition.

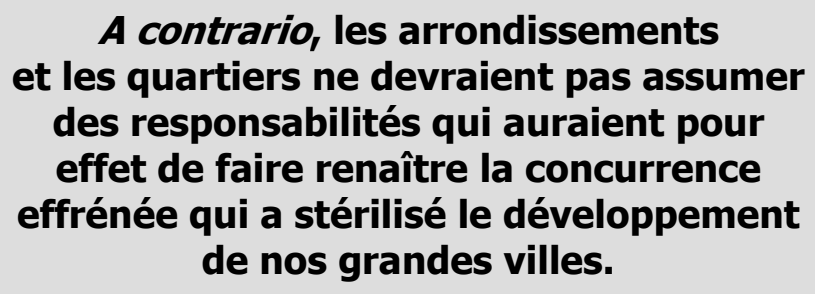

Malgré tout, il n'est pas évident que des conseils de quartier puissent se mettre en place dans tous les quartiers d'une ville. Les nouveaux quartiers urbains ne favorisent pas toujours les échanges et la vie communautaires. Le développement de ces zones résidentielles valorise plutôt un modèle à solutions individuelles. C'est par les résultats obtenus dans les quartiers plus anciens que les nouveaux quartiers découvriront l'efficacité de la voie citoyenne. Plusieurs quartiers de Québec se sont dotés d'un conseil de quartier et s'en portent très bien. Québec est toutefois la seule ville à favoriser la mise en place de conseils de quartier et l'élaboration du plan de quartier. Nous croyons que ce modèle de développement urbain pourrait aussi faire des heureux dans d'autres villes du Québec.

\section{Notes et références}

1 Yvon Leclerc est chargé de projet au siège social de l'Université du Québec et ex-président de l'Association des centres locaux de développement (CLD) du Québec. Il vient de publier, avec Claude Béland et plusieurs collaborateurs, La Voie citoyenne, pour renouveler le modèle québécois. Plusieurs chapitres de cet ouvrage traitent de la décentralisation.

2 Jacques Fiset est aujourd'hui directeur général du CLD de Québec après avoir été durant huit ans vice-président du Comité exécutif de la ville de Québec.

3 Lire à ce sujet B. Lévesque (2003), in La Voie citoyenne, pour renouveler le modèle québécois, Montréal, Éditions Plurimédia, février.

4 Cette idée a été élaborée et rodée par une urbaniste de la ville de Québec, madame Nathalie Prudhomme. 